\title{
Slant submersions in paracontact geometry
}

\author{
Yılmaz Gündüzalp (1) \\ Department of Mathematics, Dicle University, 21280, Diyarbakır, Turkey
}

\begin{abstract}
In this paper, we investigate some geometric properties of three types of slant submersions whose total space is an almost paracontact metric manifold.
\end{abstract}

Mathematics Subject Classification (2010). 53C15,53C40

Keywords. almost paracontact metric manifold, semi-Riemannian submersion, proper slant submersion

\section{Introduction}

Given a $C^{\infty}$-submersion $\psi$ from a (semi)-Riemannian manifold $\left(N, g_{N}\right)$ onto a (semi)Riemannian manifold $\left(B, g_{B}\right)$, according to the circumstances on the map $\psi:\left(N, g_{N}\right) \rightarrow$ $\left(B, g_{B}\right)$, we get the following: a (semi)-Riemannian submersion $([3,8,14,20])$, an almost Hermitian submersion ([27]), a paracontact submersion ([9]), a paracontact paracomplex submersion ([10]), a (para) quaternionic submersion $([6,17])$, a slant submersion $([12,19,22,23])$, an anti-invariant submersion $([11,24])$, a conformal semi-slant submersion $([1,13])$, a conformal anti-invariant submersion $([2])$, a hemi-slant submersion $([25])$, etc. As we know, Riemannian submersions were severally introduced by B. O'Neill ([20]) and A. Gray ([14]) in 1960s. In particular, by using the concept of almost Hermitian submersions, B. Watson ([27]) gave some differential geometric properties among fibers, base manifolds, and total manifolds. After that, there are lots of results on this issue. It is well-known that Riemannian submersions are associated with physics and have their applications in the Yang-Mills theory $([5])$, Kaluza-Klein theory $([4,15])$, supergravity and superstring theories $([16])$, etc.

The paper is organized as follows. In Section 2, we remind some concepts, which are needed in the following part. In Section 3, we study some geometric properties of three types of proper slant submersions from an almost paracontact metric manifold onto a semi-Riemannian manifold. We present examples, investigate the geometry of leaves of distributions. We obtain a necessary and sufficient circumstance for such submersions to be totally geodesic map, as well.

Email address: ygunduzalp@dicle.edu.tr Received: 07.09.2018; Accepted: 10.04.2019 


\section{Preliminaries}

\subsection{Semi-Riemannian submersions}

A $C^{\infty}$ - submersion $\psi: N \rightarrow B$ between two pseudo-Riemannian manifolds $\left(N, g_{N}\right)$ and $\left(B, g_{B}\right)$ is called a semi-Riemannian submersion if it satisfies circumstances:

(i) the fibers $\psi^{-1}(b), b \in B$, are $r$ - dimensional pseudo-Riemannian submanifolds of $N$, where $r=\operatorname{dim}(N)-\operatorname{dim}(B)$.

(ii) $\psi_{*}$ preserves scalar products of vectors normal to fibres.

The tangent bundle $T N$ of the total space $N$ has an orthogonal decomposition

$$
T N=\operatorname{ker} \psi_{*} \oplus\left(\operatorname{ker} \psi_{*}\right)^{\perp}
$$

where $k e r \psi_{*}$ is the vertical distribution while $\left(k e r \psi_{*}\right)^{\perp}$ designates the horizontal one. In ([20]), O'Neill has defined two configuration tensors $\mathcal{T}$ and $\mathcal{A}$, of the total space of a semi-Riemannian submersion by setting

$$
\mathcal{T}_{X_{1}} X_{2}=h \nabla_{v X_{1}} v X_{2}+v \nabla_{v X_{1}} h X_{2}
$$

and

$$
\mathcal{A}_{X_{1}} X_{2}=v \nabla_{h X_{1}} h X_{2}+h \nabla_{h X_{1}} v X_{2}
$$

for any $X_{1}, X_{2} \in \chi(N)$, here $v$ and $h$ are the vertical and horizontal projections respectively.

Using (2.1) and (2.2), we get

$$
\begin{gathered}
\nabla_{X_{1}} X_{2}=\mathcal{T}_{X_{1}} X_{2}+\hat{\nabla}_{X_{1}} X_{2} \\
\nabla_{X_{1}} X_{3}=\mathcal{T}_{X_{1}} X_{3}+h\left(\nabla_{X_{1}} X_{3}\right) \\
\nabla_{X_{3}} X_{1}=\mathcal{A}_{X_{3}} X_{1}+v\left(\nabla_{X_{3}} X_{1}\right) \\
\nabla_{X_{3}} X_{4}=\mathcal{A}_{X_{3}} X_{4}+h\left(\nabla_{X_{3}} X_{4}\right)
\end{gathered}
$$

for any $X_{3}, X_{4} \in \Gamma\left(\left(k e r \psi_{*}\right)^{\perp}\right), X_{1}, X_{2} \in \Gamma\left(k e r \psi_{*}\right)$. In addition, if $X_{3}$ is basic then $h\left(\nabla_{X_{1}} X_{3}\right)=h\left(\nabla_{X_{3}} X_{1}\right)=\mathcal{A}_{X_{3}} X_{1}$.

The fundamental tensor fields $\mathcal{T}, \mathcal{A}$ satisfy:

$$
\begin{gathered}
\mathcal{T}_{X_{1}} X_{2}=\mathcal{T}_{X_{2}} X_{1}, \quad X_{1}, X_{2} \in \Gamma\left(\operatorname{ker} \psi_{*}\right) ; \\
\mathcal{A}_{X_{3}} X_{4}=-\mathcal{A}_{X_{4}} X_{3}=\frac{1}{2} v\left[X_{3}, X_{4}\right], \quad X_{3}, X_{4} \in \Gamma\left(\left(k e r \psi_{*}\right)^{\perp}\right) .
\end{gathered}
$$

Lemma 2.1. If $\psi:\left(N, g_{N}\right) \rightarrow\left(B, g_{B}\right)$ is a (semi-)Riemannian submersion and $X_{3}, X_{4}$ fundamental vector fields on $N, \psi$-related to $X_{* 3}$ and $X_{* 4}$ vector fields on base manifold $B$, at that time we obtain the following features

(1) $h\left[X_{3}, X_{4}\right]$ is a fundamental vector field and $\psi_{*} h\left[X_{3}, X_{4}\right]=\left[X_{3 *}, X_{* 4}\right] \circ \psi$;

(2) $h\left(\nabla_{X_{3}} X_{4}\right)$ is a fundamental vector field $\psi$-related to $\left(\nabla_{X_{* 3}}^{*} X_{* 4}\right)$, here $\nabla$ and $\nabla^{*}$ are the Riemannian connection on $N$ and $B$;

(3) $\left[E, X_{1}\right] \in \Gamma\left(k e r \psi_{*}\right)$, for any $X_{1} \in \Gamma\left(k e r \psi_{*}\right)$ and for any fundamental vector field $E([8,21])$.

Let $\left(N, g_{N}\right)$ and $\left(B, g_{B}\right)$ be (semi-)Riemannian manifolds and $\psi:\left(N, g_{N}\right) \rightarrow\left(B, g_{B}\right)$ is a differentiable map. At that time, the second fundamental form of $\psi$ is given by

$$
\left(\nabla \psi_{*}\right)\left(X_{1}, X_{2}\right)=\nabla_{X_{1}}^{\psi} \psi_{*} X_{2}-\psi_{*}\left(\nabla_{X_{1}} X_{2}\right)
$$

for $X_{1}, X_{2} \in \Gamma(N)$, here we show conveniently by $\nabla$ the Riemannian connections of the metrics $g_{N}$ and $g_{B}$. Recall that $\psi$ is said to be harmonic if $\operatorname{trace}\left(\nabla \psi_{*}\right)=0$ and $\psi$ is called a totally geodesic map if $\left(\nabla \psi_{*}\right)\left(X_{1}, X_{2}\right)=0$ for $X_{1}, X_{2} \in \Gamma(T N)$, [18]. 


\subsection{Almost paracontact metric manifolds}

Let $N$ be a differentiable manifold of dimensional $(2 n+1)$. An almost paracontact structure on $N$ is a triple $(\varphi, \xi, \eta)$, where:

(1) $\xi$ is a Reeb vector field,

(2) $\eta$ is a one-form such that $\eta(\xi)=1$, and

(3) $\varphi$ is a tensor field of type $(1,1)$ satisfying

$$
\varphi^{2}=I d-\eta \otimes \xi, \quad \eta \circ \varphi=0, \varphi(\xi)=0 .
$$

If $N$ is equipped with a pseudo-Riemannian metric $g_{N}$ such that

$$
g_{N}\left(\varphi X_{1}, \varphi X_{2}\right)=-g_{N}\left(X_{1}, X_{2}\right)+\eta\left(X_{1}\right) \eta\left(X_{2}\right), X_{1}, X_{2} \in \chi(N),
$$

then $\left(\varphi, \xi, \eta, g_{N}\right)$ is an almost paracontact metric structure. So, the quintuple $\left(N^{2 n+1}, \varphi, \xi, \eta, g_{N}\right)$ is an almost paracontact metric manifold $([26,28])$.

Observe that, since (2.11) holds, any compatible with metric $g_{N}$ has got sign $(n+1, n)$ and by (2.10) and (2.11) we have $\eta\left(X_{1}\right)=g_{N}\left(\xi, X_{1}\right)$. Furthermore, we can determine an anti-symmetric two-form $\Phi$ by $\Phi\left(X_{1}, X_{2}\right)=g_{N}\left(X_{1}, \varphi X_{2}\right)$, which is called the fundamental 2 -form corresponding to the structure.

An almost paracontact metric structure $\left(\varphi, \xi, \eta, g_{N}\right)$ is said to be paracosymplectic, if $\nabla \eta=0$ and $\nabla \Phi=0$ are closed $([7])$, and the structure equation of a paracosymplectic manifold is given by

$$
\left(\nabla_{X_{1}} \varphi\right) X_{2}=0, \quad X_{1}, X_{2} \in \chi(N)
$$

where $\nabla$ denotes the Riemannian connection of the metric $g_{N}$ on $N$. Moreover, for a paracosymplectic manifold, we know that

$$
\nabla_{X_{1}} \xi=0 .
$$

\section{Proper slant submersions}

Let $\psi$ be a semi-Riemannian submersion from an almost paracontact metric manifold $N$ with the structure $\left(\varphi, \xi, \eta, g_{N}\right)$ onto a semi-Riemannian manifold $\left(B, g_{B}\right)$. Then for $X_{1} \in \Gamma\left(k e r \psi_{*}\right)$, we write

$$
\varphi X_{1}=\alpha X_{1}+\beta X_{1},
$$

where $\alpha X_{1}$ and $\beta X_{1}$ are vertical and horizontal parts of $\varphi X_{1}$.

In addition to for $X_{2} \in \Gamma\left(\left(k e r \psi_{*}\right)^{\perp}\right)$, we get

$$
\varphi X_{2}=t X_{2}+r X_{2},
$$

where $t X_{2}$ and $r X_{2}$ are vertical and horizontal components of $\varphi X_{2}$.

If for any spacelike or timelike vertical vector field $X_{1} \in k e r \psi_{*}-\{\xi\}$, the quotient $\frac{g_{N}\left(\alpha X_{1}, \alpha X_{1}\right)}{g_{N}\left(\varphi X_{1}, \varphi X_{1}\right)}$ is constant, i.e. it is independent of the choice of the point $p \in N$ and choice of the spacelike or timelike vertical vector field $X_{1}$ in $\operatorname{ker} \psi_{*}-\{\xi\}$, at that time we call that $\psi$ is a slant submersion. In this case, the angle $\omega$ is called the slant angle of the slant submersion.

We note that Reeb vector field $\xi$ is a spacelike vertical vector field.

Let $\left\{E_{1}, E_{2}, \xi\right\}$ be a local orthonormal frame of vertical vector fields with $g_{N}\left(E_{1}, E_{1}\right)=$ 1, i.e., such that $E_{1}$ is spacelike (if both $E_{1}$ and $E_{2}$ are timelike, the situation would be similar). From (2.11) and (3.1), we have

$$
-1=g_{N}\left(\varphi E_{1}, \varphi E_{1}\right)=g_{N}\left(\alpha E_{1}, \alpha E_{1}\right)+g_{N}\left(\beta E_{1}, \beta E_{1}\right) .
$$


On the other hand, $\alpha E_{1}=\rho E_{2}$. Let us suppose $\rho \neq 0, \pm 1$; these conditions would correspond to invariant ([9]) and anti-invariant submersions . Clearly, $\alpha E_{1}$ and $E_{2}$ have the same causal character. Depending on it and the value of $\rho$, we can separate the following three conditions:

(1) If $\alpha E_{1}$ is a timelike and $\|\rho\|>1$, at that time $g_{N}\left(\beta E_{1}, \beta E_{1}\right)=-1+\rho^{2}$ and so $\beta E_{1}$ is spacelike.

(2) If $\alpha E_{1}$ is a timelike and $\|\rho\|<1$, at that time $g_{N}\left(\beta E_{1}, \beta E_{1}\right)=-1+\rho^{2}$ and so $\beta E_{1}$ is timelike.

(3) If $\alpha E_{1}$ is a spacelike, $g_{N}\left(\beta E_{1}, \beta E_{1}\right)=-1-\rho^{2}$, and $\beta E_{1}$ is a timelike vector field.

These three conditions will correspond to three different types of proper slant submersions.

Definition 3.1. Let $\psi$ be a proper slant submersion from an almost paracontact manifold $N$ with the structure $\left(\varphi, \xi, \eta, g_{N}\right)$ onto a semi-Riemannian manifold $\left(B, g_{B}\right)$. We say that it is of

type 1 if for any spacelike (timelike) vertical vector field $X_{1} \in \Gamma\left(k e r \psi_{*}\right), \alpha X_{1}$ is timelike (spacelike), and $\frac{\left\|\alpha X_{1}\right\|}{\left\|\varphi X_{1}\right\|}>1$,

type 2 if for any spacelike (timelike) vertical vector field $X_{1} \in \Gamma\left(k e r \psi_{*}\right), \alpha X_{1}$ is timelike (spacelike), and $\frac{\left\|\alpha X_{1}\right\|}{\left\|\varphi X_{1}\right\|}<1$,

type 3 if for any spacelike (timelike) vertical vector field $X_{1} \in \Gamma\left(k e r \psi_{*}\right), \alpha X_{1}$ is timelike (spacelike).

It is known that the distribution $\left(k e r \psi_{*}\right)$ is integrable for a semi-Riemannian submersion between semi-Riemannian manifolds. In fact, its leaves are $\psi^{-1}(b), b \in B$, i.e., fibres. Thus it follows from above definition that the fibers of a slant submersion are slant submanifolds of $N$.

Theorem 3.2. Let $\psi$ be a proper slant submersion from an almost paracontact manifold $N$ with the structure $\left(\varphi, \xi, \eta, g_{N}\right)$ onto a semi-Riemannian manifold $\left(B, g_{B}\right)$. Then,

(i) $\psi$ is slant submersion of type 1 if and only if for any spacelike (timelike) vector field $X_{1} \in \Gamma\left(\mathrm{ker}_{*}\right), \alpha X_{1}$ is timelike (spacelike), and there exists a constant $\mu \in(1, \infty)$ such that

$$
\alpha^{2} X_{1}=\mu\left(X_{1}-\eta\left(X_{1}\right) \xi\right) .
$$

If $\psi$ is a proper slant submersion of type 1 , then $\mu=\cosh ^{2} \omega$, with $\omega>0$.

(ii) $\psi$ is a proper slant submersion of type 2 if and only if for any spacelike (timelike) vector field $X_{1} \in \Gamma\left(\mathrm{ker}_{*}\right), \alpha X_{1}$ is timelike (spacelike), and there exists a constant $\mu \in(0,1)$ such that

$$
\alpha^{2} X_{1}=\mu\left(X_{1}-\eta\left(X_{1}\right) \xi\right) .
$$

If $\psi$ is a proper slant submersion of type 2 , then $\mu=\cos ^{2} \omega$, with $0<\omega<2 \pi$.

(iii) $\psi$ is slant submersion of type 3 if and only if for any spacelike (timelike) vector field $X_{1} \in \Gamma\left(k e r \psi_{*}\right), \alpha X_{1}$ is timelike (spacelike), and there exists a constant $\mu \in(-\infty, 0)$ such that

$$
\alpha^{2} X_{1}=\mu\left(X_{1}-\eta\left(X_{1}\right) \xi\right) .
$$

If $\psi$ is a proper slant submersion of type 3 , then $\mu=-\sinh ^{2} \omega$, with $\omega>0$.

In every case, the angle $\omega$ is called the slant angle of the slant submersion.

Proof. (i) If $\psi$ is slant submersion of type 1, for any spacelike vertical vector field $X_{1} \in$ $\Gamma\left(k e r \psi_{*}\right), \alpha X_{1}$ is timelike, and, by virtue of (2.11), $\varphi X_{1}$ is timelike. Furthermore, they 
satisfy $\frac{\left\|\alpha X_{1}\right\|}{\left\|\varphi X_{1}\right\|}>1$. So, there exists $\omega>0$ such that

$$
\cosh \omega=\frac{\left\|\alpha X_{1}\right\|}{\left\|\varphi X_{1}\right\|}=\frac{\sqrt{-g_{N}\left(\alpha X_{1}, \alpha X_{1}\right)}}{\sqrt{-g_{N}\left(\varphi X_{1}, \varphi X_{1}\right)}} .
$$

By using (2.10), (2.11, (3.1)) and (3.6) we obtain

$$
\begin{aligned}
g_{N}\left(\alpha^{2} X_{1}, X_{1}\right) & =-g_{N}\left(\alpha X_{1}, \alpha X_{1}\right) \\
& =-\cosh ^{2} \omega g_{N}\left(\varphi X_{1}, \varphi X_{1}\right) \\
& =\cosh ^{2} \omega g_{N}\left(\varphi^{2} X_{1}, X_{1}\right) \\
& =\cosh ^{2} \omega g_{N}\left(X_{1}-\eta\left(X_{1}\right) \xi, X_{1}\right)
\end{aligned}
$$

for all $X_{1} \in \Gamma\left(k e r \psi_{*}\right)$. Since $g_{N}$ is a semi-Riemannian metric, from (3.7) we get

$$
\alpha^{2} X_{1}=\cosh ^{2} \omega\left(X_{1}-\eta\left(X_{1}\right) \xi\right), \quad X_{1} \in \Gamma\left(\operatorname{ker} \psi_{*}\right) .
$$

Let $\mu=\cosh ^{2} \omega$. Then it is obvious that $\mu \in(1, \infty)$ and $\alpha^{2}=\mu(I-\eta \otimes \xi)$.

Everything works in a similar way for any timelike vector field $X_{2} \in \Gamma\left(k e r \psi_{*}\right)$, but now, $\alpha X_{2}$ and $\varphi X_{2}$ are spacelike and hence, instead of (3.6) we can write:

$$
\cosh \omega=\frac{\left\|\alpha X_{2}\right\|}{\left\|\varphi X_{2}\right\|}=\frac{\sqrt{g_{N}\left(\alpha X_{2}, \alpha X_{2}\right)}}{\sqrt{g_{N}\left(\varphi X_{1}, \varphi X_{1}\right)}} .
$$

Since $\alpha^{2} X_{1}=\mu\left(X_{1}-\eta\left(X_{1}\right) \xi\right)$, for any spacelike or timelike $X_{1}$ we have that $\alpha^{2}=$ $\mu(I-\eta \otimes \xi)$. The converse is just a easy computation.

(ii) is obtained in a similar way.

(iii) If $\psi$ is proper slant submersion of type 3 , for any spacelike vector field $X_{1} \in$ $\Gamma\left(k e r \psi_{*}\right), \alpha X_{1}$ is spacelike, as well and hence, there exists $\omega>0$ such that

$$
\sinh \omega=\frac{\left\|\alpha X_{1}\right\|}{\left\|\varphi X_{1}\right\|}=\frac{\sqrt{g_{N}\left(\alpha X_{1}, \alpha X_{1}\right)}}{\sqrt{-g_{N}\left(\varphi X_{1}, \varphi X_{1}\right)}} .
$$

Once more, we can demonstrate that $g_{N}\left(\alpha^{2} X_{1}, X_{1}\right)=-\sinh ^{2} \omega g_{N}\left(X_{1}-\eta\left(X_{1}\right) \xi, X_{1}\right)$. Let $\mu=-\sinh ^{2} \omega$. At that time it is clear that $\mu \in(-\infty, 0)$ and $\alpha^{2}=\mu(I-\eta \otimes \xi)$.

The converse is just a easy computation.

For slant submersion of type 2, the slant angle coincides with the Wirtinger angle, i.e., the slant angle between $\varphi X_{1}$ and $\alpha X_{1}$.

Theorem 3.3. Let $\psi$ be a proper slant submersion from an almost paracontact manifold $N$ with the structure $\left(\varphi, \xi, \eta, g_{N}\right)$ onto a semi-Riemannian manifold $\left(B, g_{B}\right)$. Then,

(i) $\psi$ is slant submersion of type 1 if and only if $\alpha^{2} X_{1}=\cosh ^{2} \omega\left(X_{1}-\eta\left(X_{1}\right) \xi\right)$ for every spacelike vector field $X_{1} \in \Gamma\left(k e r \psi_{*}\right)$.

(ii) $\psi$ is slant submersion of type 2 if and only if $\alpha^{2} X_{1}=\cos ^{2} \omega\left(X_{1}-\eta\left(X_{1}\right) \xi\right)$ for every spacelike vector field $X_{1} \in \Gamma\left(k e r \psi_{*}\right)$.

Proof. (i) For every timelike vector field $X_{2} \in \Gamma\left(k e r \psi_{*}\right)$, there exists a spacelike vector field $X_{1} \in \Gamma\left(k e r \psi_{*}\right)$ such as $\alpha X_{1}=X_{2}$. Then:

$$
\alpha^{2} X_{2}=\alpha^{2} \alpha X_{1}=\alpha \alpha^{2} X_{1}=\cosh ^{2} \omega\left(\alpha X_{1}-\eta\left(\alpha X_{1}\right) \xi\right)=\cosh ^{2} \omega\left(X_{2}-\eta\left(X_{2}\right) \xi\right) .
$$

The same proof is valid for (ii), but $\alpha^{2} X_{1}=\cos ^{2} \omega\left(X_{1}-\eta\left(X_{1}\right) \xi\right)$.

Theorem 3.4. Let $\psi$ be a proper slant submersion from an almost paracontact manifold $N$ with the structure $\left(\varphi, \xi, \eta, g_{N}\right)$ onto a semi-Riemannian manifold $\left(B, g_{B}\right)$. Then $\psi$ is slant submersion of

type 1 if and only if $t \beta X_{1}=-\sinh ^{2} \omega\left(X_{1}-\eta\left(X_{1}\right) \xi\right)$ for every spacelike (timelike) vertical 
vector field $X_{1} \in \Gamma\left(\right.$ ker $\left.\psi_{*}\right)$.

type 2 if and only if $t \beta X_{1}=\sin ^{2} \omega\left(X_{1}-\eta\left(X_{1}\right) \xi\right)$ for every spacelike (timelike) vertical vector field $X_{1} \in \Gamma\left(k e r \psi_{*}\right)$.

type 3 if and only if $t \beta X_{1}=\cosh ^{2} \omega\left(X_{1}-\eta\left(X_{1}\right) \xi\right)$ for every spacelike (timelike) vertical vector field $X_{1} \in \Gamma\left(\operatorname{ker} \psi_{*}\right)$.

Proof. For any vertical vector field $X_{1} \in \Gamma\left(k e r \psi_{*}\right)$, it holds

$$
X_{1}-\eta\left(X_{1}\right) \xi=\varphi^{2} X_{1}=\alpha^{2} X_{1}+\beta \alpha X_{1}+t \beta X_{1}+r \beta X_{1} .
$$

Equalizing the vertical and the horizontal parts of the above equation, we obtain:

$$
\alpha^{2} X_{1}+t \beta X_{1}=X_{1}-\eta\left(X_{1}\right) \xi, \quad \beta \alpha X_{1}+r \beta X_{1}=0 .
$$

Hence, for a slant submersion of type 1 ,

$$
t \beta X_{1}=X_{1}-\eta\left(X_{1}\right) \xi-\alpha^{2} X_{1}=\left(1-\cosh ^{2} \omega\right)\left(X_{1}-\eta\left(X_{1}\right) \xi\right)=-\sinh ^{2} \omega\left(X_{1}-\eta\left(X_{1}\right) \xi\right),
$$

while for a slant submersion of type 2 ,

$$
t \beta X_{1}=X_{1}-\eta\left(X_{1}\right) \xi-\alpha^{2} X_{1}=\left(1-\cos ^{2} \omega\right)\left(X_{1}-\eta\left(X_{1}\right) \xi\right)=\sin ^{2} \omega\left(X_{1}-\eta\left(X_{1}\right) \xi\right),
$$

and, for a slant submersion of type 3 ,

$$
t \beta X_{1}=X_{1}-\eta\left(X_{1}\right) \xi-\alpha^{2} X_{1}=\left(1+\sinh ^{2} \omega\right)\left(X_{1}-\eta\left(X_{1}\right) \xi\right)=\cosh ^{2} \omega\left(X_{1}-\eta\left(X_{1}\right) \xi\right) .
$$

The converse results are deduced from the same equations.

Theorem 3.5. Let $\psi$ be a semi-Riemannian submersion from an almost paracontact metric manifold $\left(N_{2 n}^{4 n+1}, \varphi, \eta, \xi, g_{N}\right)$ onto a semi-Riemannian manifold $\left(B_{n}^{2 n}, g_{B}\right)$. Then $\psi$ is a slant submersion of

type 1 if and only if $r^{2} X_{2}=\cosh ^{2} \omega X_{2}$ for every spacelike (timelike) horizontal vector field $X_{2} \in \Gamma\left(\left(k e r \psi_{*}\right)^{\perp}\right)$.

type 2 if and only if $r^{2} X_{2}=\cos ^{2} \omega X_{2}$ for every spacelike (timelike) horizontal vector field $X_{2} \in \Gamma\left(\left(\operatorname{ker} \psi_{*}\right)^{\perp}\right)$.

Proof. In the case of a slant submersion of

type 1 , for every horizontal timelike (spacelike) vector field $X_{2} \in \Gamma\left(\left(k e r \psi_{*}\right)^{\perp}\right)$, there exists a spacelike (timelike) vertical vector field $X_{1} \in \Gamma\left(k e r \psi_{*}\right)$ such as $\beta X_{1}=X_{2}$. From (3.9), we obtain

$$
r^{2} X_{2}=r^{2} \beta X_{1}=-r \beta \alpha X_{1}=\beta \alpha^{2} X_{1}=\beta\left(\cosh ^{2} \omega\left(X_{1}-\eta\left(X_{1}\right) \xi\right)\right) .
$$

From (3.10), we get $r^{2} X_{2}=\cosh ^{2} \omega\left(\beta X_{1}-\eta\left(\beta X_{1}\right) \xi\right)$. Since $\beta X_{1} \perp \xi$, we obtain $\eta\left(\beta X_{1}\right)=0$ and thus $r^{2} X_{2}==\cosh ^{2} \omega X_{2}$.

In the case of a slant submersion of type 2 , in a similar way, we get

$$
r^{2} X_{2}=\cos ^{2} \omega X_{2} .
$$

The converse results follow from the fact that $t\left(\left(\operatorname{ker} \psi_{*}\right)^{\perp}\right)=\left(\operatorname{ker} \psi_{*}\right) \oplus\langle\xi\rangle$.

Theorem 3.6. Let $\psi$ be a semi-Riemannian submersion from an almost paracontact metric manifold $\left(N_{2 n}^{4 n+1}, \varphi, \eta, \xi, g_{N}\right)$ onto a semi-Riemannian manifold $\left(B_{2 j}^{2 n}, g_{B}\right)(0<j<n)$. At that time, $\psi$ is a slant submersion of type 3 if and only if $r^{2} X_{2}=-\sinh ^{2} \omega X_{2}$ for every horizontal vector field $X_{2} \in \Gamma\left(\left(k e r \psi_{*}\right)^{\perp}\right)$.

Proof. If $X_{1}$ is a spacelike (timelike) vertical vector field, $\alpha X_{1}$ is also spacelike (timelike) and $\beta X_{1}$ is timelike (spacelike). Therefore, given that the dimension of $B$ is half the dimension of $N$, if $X_{2}$ is a timelike (spacelike) horizontal vector field, then there exists a vertical vector field $X_{1} \in \Gamma\left(k e r \psi_{*}\right)$ such that $\beta X_{1}=X_{2}$. Then, from (3.10) we have $r^{2} X_{2}=\beta\left(-\sinh ^{2} \omega\left(X_{1}-\eta\left(X_{1}\right) \xi\right)\right)=-\sinh ^{2} \omega X_{2}$. The converse results follow from the fact that $t\left(\left(k e r \psi_{*}\right)^{\perp}\right)=\left(k e r \psi_{*}\right) \oplus\langle\xi\rangle$. 
From Theorem 3.2, (2.11) and (3.1) we obtain the following result.

Lemma 3.7. Let $\psi$ be a semi-Riemannian submersion from an almost paracontact metric manifold $\left(N, \varphi, \eta, \xi, g_{N}\right)$ onto a semi-Riemannian manifold $\left(B, g_{B}\right)$.

If $\psi$ is a proper slant submersion of type 1 , then, for any spacelike (timelike) vector fields $X_{1}, X_{2} \in \Gamma\left(\operatorname{ker} \psi_{*}\right)$, we have

$$
\begin{aligned}
& g_{N}\left(\alpha X_{1}, \alpha X_{2}\right)=\cosh ^{2} \omega\left(-g_{N}\left(X_{1}, X_{2}\right)+\eta\left(X_{1}\right) \eta\left(X_{2}\right)\right) \\
& g_{N}\left(\beta X_{1}, \beta X_{2}\right)=-\sinh ^{2} \omega\left(-g_{N}\left(X_{1}, X_{2}\right)+\eta\left(X_{1}\right) \eta\left(X_{2}\right)\right)
\end{aligned}
$$

If $\psi$ is a proper slant submersion of type 2 , then, for any spacelike (timelike) vector fields $X_{1}, X_{2} \in \Gamma\left(\operatorname{ker} \psi_{*}\right)$, we have

$$
\begin{aligned}
& g_{N}\left(\alpha X_{1}, \alpha X_{2}\right)=\cos ^{2} \omega\left(-g_{N}\left(X_{1}, X_{2}\right)+\eta\left(X_{1}\right) \eta\left(X_{2}\right)\right) \\
& g_{N}\left(\beta X_{1}, \beta X_{2}\right)=\sin ^{2} \omega\left(-g_{N}\left(X_{1}, X_{2}\right)+\eta\left(X_{1}\right) \eta\left(X_{2}\right)\right) .
\end{aligned}
$$

If $\psi$ is a proper slant submersion of type 3 , then, for any spacelike (timelike) vector fields $X_{1}, X_{2} \in \Gamma\left(k e r \psi_{*}\right)$, we have

$$
\begin{aligned}
& g_{N}\left(\alpha X_{1}, \alpha X_{2}\right)=-\sinh ^{2} \omega\left(-g_{N}\left(X_{1}, X_{2}\right)+\eta\left(X_{1}\right) \eta\left(X_{2}\right)\right) \\
& g_{N}\left(\beta X_{1}, \beta X_{2}\right)=\cosh ^{2} \omega\left(-g_{N}\left(X_{1}, X_{2}\right)+\eta\left(X_{1}\right) \eta\left(X_{2}\right)\right) .
\end{aligned}
$$

Note that given a semi-Euclidean space $R_{n}^{2 n+1}$ with coordinates $\left(x_{1}, \ldots, x_{2 n}, z\right)$ on $R_{n}^{2 n+1}$, we can naturally choose an almost paracontact structure $(\varphi, \xi, \eta)$ on $R_{n}^{2 n+1}$ as follows:

$$
\eta=d z, \quad \xi=\frac{\partial}{\partial z}, \varphi\left(\frac{\partial}{\partial x_{2 i}}\right)=\frac{\partial}{\partial x_{2 i-1}}, \varphi\left(\frac{\partial}{\partial x_{2 i-1}}\right)=\frac{\partial}{\partial x_{2 i}}, \varphi(\xi)=0
$$

where $i=1, \ldots, n$. Let $R_{n}^{2 n+1}$ be a semi-Euclidean space of signature $(+,-,+,-, \ldots,+)$ with respect to the canonical basis $\left(\frac{\partial}{\partial x_{1}}, \ldots, \frac{\partial}{\partial x_{2 n}}, \frac{\partial}{\partial z}\right)$.

Now, we can present four examples of proper slant submersions.

Example 3.8. Determine a map $\psi: R_{2}^{5} \rightarrow R_{1}^{2}$ by

$$
\psi\left(x_{1}, x_{2}, x_{3}, x_{4}, z\right)=\left(\frac{x_{1}-x_{3}}{\sqrt{2}}, x_{2}\right) .
$$

At that time, by direct calculations we obtain

$$
k e r \psi_{*}=\operatorname{span}\left\{U_{1}=\frac{\partial}{\partial x_{1}}+\frac{\partial}{\partial x_{3}}, U_{2}=\frac{\partial}{\partial x_{4}}, U_{3}=\xi=\frac{\partial}{\partial z}\right\}
$$

and

$$
\left(k e r \psi_{*}\right)^{\perp}=\operatorname{span}\left\{X_{1}=\frac{\partial}{\partial x_{1}}-\frac{\partial}{\partial x_{3}}, X_{2}=\frac{\partial}{\partial x_{2}}\right\} .
$$

Thus, the map $\psi$ is a slant submersion of type 2 with the slant angle $\omega$ with $\cos ^{-1}\left(\frac{1}{\sqrt{2}}\right)$.

Example 3.9. Define a map $\psi: R_{2}^{5} \rightarrow R_{1}^{2}$ by

$$
\psi\left(x_{1}, x_{2}, x_{3}, x_{4}, z\right)=\left(x_{2} \sinh x+x_{3} \cosh x, x_{1} \sinh y+x_{4} \cosh y\right),
$$

any for $x, y \in R$. Then, by direct calculations we get

$k e r \psi_{*}=\operatorname{span}\left\{U_{1}=\cosh x \frac{\partial}{\partial x_{2}}-\sinh x \frac{\partial}{\partial x_{3}}, U_{2}=\cosh y \frac{\partial}{\partial x_{1}}-\sinh y \frac{\partial}{\partial x_{4}}, U_{3}=\xi=\frac{\partial}{\partial z}\right\}$ and

$$
\left(k e r \psi_{*}\right)^{\perp}=\operatorname{span}\left\{X_{1}=-\sinh x \frac{\partial}{\partial x_{2}}+\cosh x \frac{\partial}{\partial x_{3}}, X_{2}=\sinh y \frac{\partial}{\partial x_{1}}-\cosh y \frac{\partial}{\partial x_{4}}\right\} .
$$

Thus, the map $\psi$ is a slant submersion of type 1 with the slant angle $\cosh \omega=\cosh (x-y)$. 
Example 3.10. Define a map $\psi: R_{2}^{5} \rightarrow R_{1}^{2}$ by

$$
\psi\left(x_{1}, x_{2}, x_{3}, x_{4}, z\right)=\left(x_{1} \sin x+x_{3} \cos x, x_{2} \sin y+x_{4} \cos y\right),
$$

for any $x, y \in R$. The map $\psi$ is a slant submersion of type 2 with the slant angle $\cos \omega=$ $\cos (x-y)$.

Example 3.11. Define a map $\psi: R_{2}^{5} \rightarrow R_{1}^{2}$ by

$$
\psi\left(x_{1}, x_{2}, x_{3}, x_{4}, z\right)=\left(x_{2} \cosh x+x_{3} \sinh x, x_{4}\right),
$$

for any $x \in R^{+}$. The map $\psi$ is a slant submersion of type 3 with the slant angle $\alpha^{2}=$ $-\sinh ^{2} x$.

Let $\psi$ be proper slant submersions of type 1,2 and 3 from a paracosymplectic manifold $N$ with the structure $\left(g_{N}, \varphi, \eta, \xi\right)$ onto a semi-Riemannian manifold $\left(B, g_{B}\right)$. From $(2.11),(3.1)$ and (3.2), one can simply see that

$$
g_{N}\left(X_{1}, \alpha X 2\right)=-g_{N}\left(\alpha X_{1}, X_{2}\right)
$$

and

$$
g_{N}\left(\beta X_{1}, X_{3}\right)=-g_{N}\left(X_{1}, t X_{3}\right),
$$

for spacelike (timelike) vector fields $X_{1}, X_{2} \in \Gamma\left(k e r \psi_{*}\right), X_{3} \in \Gamma\left(\left(k e r \psi_{*}\right)^{\perp}\right)$.

Using (2.3),(2.5) and (2.13) we have

$$
\mathcal{T}_{X_{1}} \xi=0, \mathcal{A}_{X_{3}} \xi=0
$$

for spacelike (timelike) vector fields $X_{1} \in \Gamma\left(k e r \psi_{*}\right), X_{3} \in \Gamma\left(k e r \psi_{*}\right)^{\perp}$.

We determine the covariant derivatives of $\alpha$ and $\beta$ as follows

$$
\left(\nabla_{X_{1}} \alpha\right) X_{2}=\hat{\nabla}_{X_{1}} \alpha X_{2}-\alpha \hat{\nabla}_{X_{1}} X_{2}
$$

and

$$
\left(\nabla_{X_{1}} \beta\right) Y=h \nabla_{X_{1}} \beta X_{2}-\beta \hat{\nabla}_{X_{1}} X_{2}
$$

for spacelike (timelike) vector fields $X_{1}, X_{2} \in \Gamma\left(k e r F_{*}\right)$, where $\hat{\nabla}_{X_{1}} X_{2}=v \nabla_{X_{1}} X_{2}$. Then we easily have

Lemma 3.12. Let $\left(N, g_{N}, \varphi, \eta, \xi\right)$ be a paracosymplectic manifold and $\left(B, g_{B}\right)$ a semiRiemannian manifold. Let $\psi: N \rightarrow B$ be proper slant submersions of type 1, 2 and 3 . Then, we have

$$
\begin{aligned}
\hat{\nabla}_{X_{1}} \alpha X_{2}+\mathcal{T}_{X_{1}} \beta X_{2} & =\alpha \hat{\nabla}_{X_{1}} X_{2}+t \mathcal{T}_{X_{1} X_{2}} \\
\mathcal{T}_{X_{1}} \alpha X_{2}+h \nabla_{X_{1}} \beta X_{2} & =\beta \hat{\nabla}_{X_{1}} X_{2}+r \mathcal{T}_{X_{1}} X_{2}
\end{aligned}
$$

for any spacelike(timelike)vector fields $X_{1}, X_{2} \in \Gamma\left(k e r \psi_{*}\right)$.

We say that $\beta$ is parallel with respect to the Riemannian connection $\nabla$ on $\left(k e r \psi_{*}\right)$ if its covariant derivative with respect to $\nabla$ vanishes, i.e., we get

$$
\left(\nabla_{X_{1}} \beta\right) X_{2}=h \nabla_{X_{1}} \beta X_{2}-\beta \hat{\nabla}_{X_{1}} X_{2}=0
$$

for any spacelike (timelike) vertical vector fields $X_{1}, X_{2} \in \Gamma\left(k e r \psi_{*}\right)$.

Theorem 3.13. Let $\psi$ be a proper slant submersions of type 1,2 and 3 from a paracosymplectic manifold $\left(N, g_{N}, \varphi, \eta, \xi\right)$ onto a semi-Riemannian manifold $\left(B, g_{B}\right)$. At that time, the fibres are not proper totally umbilical.

Proof. See [19].

We now indicate the orthogonal complementary distribution to $\beta\left(k e r \psi_{*}\right)$ in $\left(k e r \psi_{*}\right)^{\perp}$ by $\tau$. At that time, we obtain the following. 
Theorem 3.14. Let $\psi:\left(N, g_{N}, \varphi, \eta, \xi\right) \rightarrow\left(B, g_{B}\right)$ be a proper slant submersions of type of 1,2 and 3 . If $N$ is a paracosymplectic manifold, then $\tau$ is an invariant distribution of $\left(k e r \psi_{*}\right)^{\perp}$, with respect to $\varphi$.

Proof. For $X_{2} \in \Gamma(\tau)$, using (2.11) and (3.1), we get

$$
\begin{aligned}
g_{N}\left(\varphi X_{2}, \beta X_{1}\right) & =-g_{N}\left(X_{2}, X_{1}\right)-g_{N}\left(\varphi X_{2}, \alpha X_{1}\right)+\eta\left(X_{1}\right) \eta\left(X_{2}\right) \\
& =-g_{N}\left(\varphi X_{2}, \alpha X_{1}\right) \\
& =g_{N}\left(X_{2}, \varphi \alpha X_{1}\right)=0
\end{aligned}
$$

for any spacelike (timelike) vector field $X_{1} \in \Gamma\left(k e r \psi_{*}\right)$.

In a similar way, we have $g_{N}\left(\varphi X_{2}, X_{3}\right)=-g_{N}\left(X_{2}, \varphi X_{3}\right)=0$ due to $\varphi X_{3} \in \Gamma\left(\left(k e r \psi_{*}\right) \oplus\right.$ $\left.\beta\left(k e r \psi_{*}\right)\right)$ for any spacelike (timelike) vector field $X_{2} \in \Gamma(\tau)$ and $X_{3} \in \Gamma\left(k e r \psi_{*}\right)$. Hence, proof is complete.

Corollary 3.15. Let $\psi:\left(N_{2 n}^{4 n+1}, g_{N}, \varphi, \eta, \xi\right) \rightarrow\left(B_{2 j}^{2 n}, g_{B}\right)(0<j<n)$ be a proper slant submersion of type 3 . If $N$ is a paracosymplectic manifold and $\left\{E_{1}, \ldots, E_{2 n}, \xi\right\}$ is a local orthonormal basis of $\left(k e r \psi_{*}\right)$, at that time $\left\{\frac{1}{\cosh \omega} \beta E_{1}, \ldots, \frac{1}{\cosh \omega} \beta E_{2 n}\right\}$ is a local orthonormal basis of $\beta\left(\right.$ ker $\left.\psi_{*}\right)$.

Proof. It will be enough to demonstrate that $g_{N}\left(\frac{1}{\cosh \omega} \beta E_{i}, \frac{1}{\cosh \omega} \beta E_{j}\right)=\epsilon_{i} \delta_{i j}$, for any $i, j \in\{1, \ldots, n\}$, where $\epsilon_{i}=\operatorname{sgn}\left(g_{N}\left(E_{1}, E_{1}\right)\right)= \pm 1$. By using (3.16), we get

$$
g_{N}\left(\frac{1}{\cosh \omega} \beta E_{i}, \frac{1}{\cosh \omega} \beta E_{j}\right)=\left(\frac{1}{\cosh \omega}\right)^{2} \cosh ^{2} \omega g_{N}\left(E_{i}, E_{j}\right)=\epsilon_{i} \delta_{i j},
$$

which proves the assertion.

In a similar way, we get the following.

Lemma 3.16. Let $\psi:\left(N_{2 n}^{4 n+1}, g_{N}, \varphi, \eta, \xi\right) \rightarrow\left(B_{2 j}^{2 n}, g_{B}\right)(0<j<n)$ be a proper slant submersion of type 3 . If $N$ is a paracosymplectic manifold and $E_{1}, \ldots, E_{n}, \xi$ are orthogonal unit vector fields in $\left(k e r \psi_{*}\right)$, then

is a local orthonormal basis of $\left(\right.$ ker $\left.\psi_{*}\right)$.

$$
\left\{E_{1}, \frac{1}{\sinh \omega} \alpha E_{1}, E_{2}, \frac{1}{\sinh \omega} \alpha E_{2}, \ldots, E_{n}, \frac{1}{\sinh \omega} \alpha E_{n}, \xi\right\}
$$

Let $\psi$ be a proper slant submersion of type 3 from a paracosymplectic manifold $\left(N^{4 n+1}, g_{N}, \varphi, \eta, \xi\right)$ onto a semi-Riemannian manifold $\left(B^{2 n}, g_{B}\right)$. We call such an orthonormal frame

$$
\left\{E_{1}, \frac{1}{\sinh \omega} \alpha E_{1}, E_{2}, \frac{1}{\sinh \omega} \alpha E_{2}, \ldots, E_{2 n}, \frac{1}{\sinh \omega} \alpha E_{n}, \frac{1}{\cosh \omega} \beta E_{1}, \ldots, \frac{1}{\cosh \omega} \beta E_{2 n}\right\}
$$

an adapted slant frame for proper slant submersion of type 3 .

Proposition 3.17. Let $\psi:\left(N, g_{N}, \varphi, \eta, \xi\right) \rightarrow\left(B, g_{B}\right)$ be a proper slant submersion of type 1. If $N$ is a paracosymplectic manifold and $\beta$ is parallel with respect to $\nabla$ on $\left(k e r \psi_{*}\right)$, then we have

$$
\mathcal{T}_{\alpha X_{1}} \alpha X_{1}=\cosh ^{2} \omega \mathcal{T}_{X_{1}} X_{1}
$$

for any spacelike (timelike) vector field $X_{1} \in \Gamma\left(k e r \psi_{*}\right)$.

Proof. If $\beta$ is parallel, at that time from Lemma 3.12 we get $r \mathcal{T}_{X_{1}} X_{2}=\mathcal{T}_{X_{1}} \alpha X_{2}$ for any spacelike (timelike) vector fields $X_{1}, X_{2} \in \Gamma\left(k e r \psi_{*}\right)$. Interchanging the role of $X_{1}$ and $X_{2}$, we get $r \mathcal{T}_{X_{2}} X_{1}=\mathcal{T}_{X_{2}} \alpha X_{1}$. Thus we have

$$
r \mathcal{T}_{X_{1}} X_{2}-r \mathcal{T}_{X_{2}} X_{1}=\mathcal{T}_{X_{1}} \alpha X_{2}-\mathcal{T}_{X_{2}} \alpha X_{1}
$$

Since $\mathcal{T}$ is symmetric, we derive $\mathcal{T}_{X_{1}} \alpha X_{2}=\mathcal{T}_{X_{2}} \alpha X_{1}$. Then substituting $X_{2}$ by $\alpha X_{1}$ we get $\mathcal{T}_{X_{1}} \alpha^{2} X_{1}=\mathcal{T}_{\alpha X_{1}} \alpha X_{1}$. Using (3.3) and (3.19) we obtain (3.23).

In a similar way, we obtain the following. 
Corollary 3.18. Let $\psi:\left(N, g_{N}, \varphi, \eta, \xi\right) \rightarrow\left(B, g_{B}\right)$ be a proper slant submersion of type 2. If $N$ is a paracosymplectic manifold and $\beta$ is parallel with respect to $\nabla$ on (ker $\left.\psi_{*}\right)$, then we have

$$
\mathcal{T}_{\alpha X_{1}} \alpha X_{1}=\cos ^{2} \omega \mathcal{T}_{X_{1}} X_{1}
$$

for any spacelike (timelike) vector field $X_{1} \in \Gamma\left(k e r \psi_{*}\right)$.

Corollary 3.19. Let $\psi:\left(N, g_{N}, \varphi, \eta, \xi\right) \rightarrow\left(B, g_{B}\right)$ be a proper slant submersion of type 3. If $N$ is a paracosymplectic manifold and $\beta$ is parallel with respect to $\nabla$ on $\left(k e r \psi_{*}\right)$, then we have

$$
\mathcal{T}_{\alpha X_{1}} \alpha X_{1}=-\sinh ^{2} \omega \mathcal{T}_{X_{1}} X_{1}
$$

for any spacelike (timelike) vector field $X_{1} \in \Gamma\left(k e r \psi_{*}\right)$.

Theorem 3.20. Let $\psi:\left(N_{2 n}^{4 n+1}, g_{N}, \varphi, \eta, \xi\right) \rightarrow\left(B_{2 j}^{2 n}, g_{B}\right)(0<j<n)$ be a proper slant submersion of type 3 . If $N$ is a paracosymplectic manifold and $\beta$ is parallel with respect to $\nabla$ on $\left(k e r \psi_{*}\right)$, at that time $\psi$ is a harmonic map.

Proof. Using (2.9), we obtain

$$
\left(\nabla \psi_{*}\right)\left(X_{3}, X_{4}\right)=0
$$

for any spacelike (timelike) vector fields $X_{3}, X_{4} \in\left(k e r \psi_{*}\right)^{\perp}$. A proper slant submersion of type $3 \psi$ is harmonic if and only if $\sum_{i=1}^{2 n}\left(\nabla \psi_{*}\right)\left(E_{i}^{*}, E_{i}^{*}\right)=\sum_{i=1}^{2 n}\left(\nabla \psi_{*}\right)\left(\mathcal{T}_{E_{i}^{*}} E_{i}^{*}\right)=0$, here $\left\{E_{i}^{*}\right\}_{i=1}^{2 n}$ is an orthonormal basis of $\left(k e r \psi_{*}\right)$. Hence, using Lemma 3.16 we should write

$$
\kappa=-\sum_{i=1}^{n} \psi_{*}\left(\mathcal{T}_{E_{i}} E_{i}+\mathcal{T}_{\frac{1}{\sinh \omega}} \alpha E_{i} \frac{1}{\sinh \omega} \alpha E_{i}\right)-\mathcal{T}_{\xi} \xi
$$

From (3.19), we have

$$
\kappa=-\sum_{i=1}^{n} \psi_{*}\left(\mathcal{T}_{E_{i}} E_{i}+\frac{1}{\sinh ^{2} \varphi} \mathcal{T}_{\alpha E_{i}} \alpha E_{i}\right)
$$

Then using (3.25) we have

$$
\kappa=-\sum_{i=1}^{n} \psi_{*}\left(\mathcal{T}_{E_{i}} E_{i}-\mathcal{T}_{E_{i}} E_{i}\right)=0
$$

which shows that $\psi$ is harmonic.

Putting $\theta=\alpha^{2}$, we define $\nabla \theta$ by

$$
\left(\nabla_{X_{1}} \theta\right) X_{2}=v \nabla_{X_{1}} \theta X_{2}-\theta \hat{\nabla}_{X_{1}} X_{2}
$$

for any spacelike(timelike) vector fields $X_{1}, X_{2} \in \Gamma\left(k e r \psi_{*}\right)$.

Theorem 3.21. Let $\psi:\left(N, g_{N}, \varphi, \eta, \xi\right) \rightarrow\left(B, g_{B}\right)$ be a proper slant submersion of type 1 . If $N$ is a paracosymplectic manifold, then $\nabla \theta=0$.

Proof. Using (3.3), we have

$$
\theta \hat{\nabla}_{X_{1}} X_{2}=\cosh ^{2} \omega\left(\hat{\nabla}_{X_{1}} X_{2}-\eta\left(\hat{\nabla}_{X_{1}} X_{2}\right) \xi\right)
$$

for all spacelike(timelike) vector fields $X_{1}, X_{2} \in \Gamma\left(k e r \psi_{*}\right)$. On the other hand, from (3.3) and (2.13) we obtain

$$
\begin{aligned}
v\left(\hat{\nabla}_{X_{1}} \theta X_{2}\right) & =\cosh ^{2} \omega\left(\hat{\nabla}_{X_{1}} X_{2}-\left(\hat{\nabla}_{X_{1}} \eta\left(X_{2}\right)\right) \xi\right) \\
& =\cosh ^{2} \omega\left(\hat{\nabla}_{X_{1}} X_{2}-\eta\left(\hat{\nabla}_{X_{1}} X_{2}\right)-g_{N}\left(X_{2}, \nabla_{X_{1}} \xi\right)\right) \\
& =\cosh ^{2} \omega\left(\hat{\nabla}_{X_{1}} X_{2}-\eta\left(\hat{\nabla}_{X_{1}} X_{2}\right) .\right.
\end{aligned}
$$

Using (3.27) and (3.28), we get $\left(\nabla_{X_{1}} \theta\right) X_{2}=0$.

Now, we examine the geometry of the leaves of the distributions $\left(k e r \psi_{*}\right)$ and $\left(k e r \psi_{*}\right)^{\perp}$. 
Theorem 3.22. Let $\psi:\left(N, g_{N}, \varphi, \eta, \xi\right) \rightarrow\left(B, g_{B}\right)$ be a proper slant submersion of type 1 . If $N$ is a paracosymplectic manifold, then the distribution $\left(k e r \psi_{*}\right)$ defines a totally geodesic foliation on $N$ if and only if

$$
g_{N}\left(h \nabla_{X_{1}} \beta \alpha X_{2}, X_{3}\right)=g_{N}\left(h \nabla_{X_{1}} \beta X_{2}, r X_{3}\right)+g_{N}\left(\mathcal{T}_{X_{1}} \beta X_{2}, t X_{3}\right)
$$

for spacelike (timelike) vector fields $X_{1}, X_{2} \in \Gamma\left(k e r \psi_{*}\right)$ and $X_{3} \in \Gamma\left(\left(k e r \psi_{*}\right)^{\perp}\right)$.

Proof. For spacelike (timelike) vector fields $X_{1}, X_{2} \in \Gamma\left(k e r \psi_{*}\right)$ and $X_{3} \in \Gamma\left(\left(k e r \psi_{*}\right)^{\perp}\right)$, since (2.10) and (2.12) we obtain

$$
g_{N}\left(\nabla_{X_{1}} X_{2}, X_{3}\right)=g_{N}\left(\varphi \nabla_{X_{1}} \varphi X_{2}, X_{3}\right)+g_{N}\left(\nabla_{X_{1}} X_{2}, \xi\right) \eta\left(X_{3}\right)
$$

Using (3.1) and (3.2) we get

$$
\begin{aligned}
g_{N}\left(\nabla_{X_{1}} X_{2}, X_{3}\right) & =g_{N}\left(\nabla_{X_{1}} \alpha^{2} X_{2}, X 3\right)+g_{N}\left(\nabla_{X_{1}} \beta \alpha X_{2}, X_{3}\right) \\
& -g_{N}\left(\nabla_{X_{1}} \beta X_{2}, t X_{3}\right)-g_{N}\left(\nabla_{X_{1}} \beta X_{2}, r X_{3}\right) .
\end{aligned}
$$

Then from (2.4),(2.13) and (3.3) we obtain

$$
\begin{aligned}
g_{N}\left(\nabla_{X_{1}} X_{2}, X_{3}\right) & =\cosh ^{2} \omega g_{N}\left(\nabla_{X_{1}} X_{2}, X_{3}\right)+g_{N}\left(h \nabla_{X_{1}} \beta \alpha X_{2}, X_{3}\right) \\
& -g_{N}\left(\mathcal{T}_{X_{1}} \beta X_{2}, t X_{3}\right)-g_{N}\left(h \nabla_{X_{1}} \beta X_{2}, r X_{3}\right) .
\end{aligned}
$$

Hence we have

$$
\begin{aligned}
-\sinh ^{2} \omega g_{N}\left(\nabla_{X_{1}} X_{2}, X_{3}\right) & =g_{N}\left(h \nabla_{X_{1}} \beta \alpha X_{2}, X_{3}\right) \\
& -g_{N}\left(\mathcal{T}_{X_{1}} \beta X_{2}, t X_{3}\right)-g_{N}\left(h \nabla_{X_{1}} \beta X_{2}, r X_{3}\right)
\end{aligned}
$$

which proves assertion.

Theorem 3.23. Let $\psi:\left(N, g_{N}, \varphi, \eta, \xi\right) \rightarrow\left(B, g_{B}\right)$ be a proper slant submersion of type 1. If $N$ is a paracosymplectic manifold, then the distribution $\left(k e r \psi_{*}\right)^{\perp}$ defines a totally geodesic foliation on $N$ if and only if

$$
g_{N}\left(h \nabla_{X_{1}} X_{2}, \beta \alpha X_{3}\right)=g_{N}\left(\mathcal{A}_{X_{1}} t X_{2}+h \nabla_{X_{1}} r X_{2}, \beta X_{3}\right)
$$

for any spacelike (timelike)vector fields $X_{3} \in \Gamma\left(k e r \psi_{*}\right)$ and $X_{1}, X_{2} \in \Gamma\left(\left(k e r \psi_{*}\right)^{\perp}\right)$.

Proof. For $X_{3} \in \Gamma\left(k e r \psi_{*}\right)$ and $X_{1}, X_{2} \in \Gamma\left(\left(k e r \psi_{*}\right)^{\perp}\right)$, from (2.12) and (3.1) we obtain

$$
\begin{aligned}
g_{N}\left(\nabla_{X_{1}} X_{2}, X_{3}\right) & =-g_{N}\left(\nabla_{X_{1}} \varphi X_{2}, \varphi X_{3}\right)+g_{N}\left(\nabla_{X_{1}} X_{2}, \xi\right) \eta\left(X_{3}\right) \\
& =-g_{N}\left(\varphi \nabla_{X_{1}} X_{2}, \alpha X_{3}\right)-g_{N}\left(\nabla_{X_{1}} \varphi X_{2}, \beta X_{3}\right) \\
& +g_{N}\left(\nabla_{X_{1}} X_{2}, \xi\right) \eta\left(X_{3}\right) .
\end{aligned}
$$

Using (3.1) in (3.31), we get

$$
\begin{aligned}
g_{N}\left(\nabla_{X_{1}} X_{2}, X_{3}\right) & =g_{N}\left(\nabla_{X_{1}} X_{2}, \alpha^{2} X_{3}\right)+g_{N}\left(\nabla_{X_{1}} X_{2}, \beta \alpha X_{3}\right) \\
& -g_{N}\left(\nabla_{X_{1}} \varphi X_{2}, \beta X_{3}\right)+g_{N}\left(\nabla_{X_{1}} X_{2}, \xi\right) \eta\left(X_{3}\right) .
\end{aligned}
$$

Using (3.2) and (3.3) we get

$$
\begin{aligned}
g_{N}\left(\nabla_{X_{1}} X_{2}, X_{3}\right) & =\cosh ^{2} \omega g_{N}\left(\nabla_{X_{1}} X_{2}, X_{3}\right)-\cosh ^{2} \omega \eta\left(\nabla_{X_{1}} X_{2}\right) \eta\left(X_{3}\right) \\
& +g_{N}\left(\nabla_{X_{1}} X_{2}, \beta \alpha X_{3}\right)-g_{N}\left(\nabla_{X_{1}} t X_{2}, \beta X_{3}\right) \\
& -g_{N}\left(\nabla_{X_{1}} r X_{2}, \beta X_{3}\right)+g_{N}\left(\nabla_{X_{1}} X_{2}, \xi\right) \eta\left(X_{3}\right) .
\end{aligned}
$$

Using (2.5),(2.6) and (2.13) in (3.33), we get

$$
-\sinh ^{2} \omega g_{N}\left(\nabla_{X_{1}} X_{2}, X_{3}\right)=g_{N}\left(h \nabla_{X_{1}} X_{2}, \beta \alpha X_{3}\right)-g_{N}\left(\mathcal{A}_{X_{1}} t X_{2}+h \nabla_{X_{1}} r X_{2}, \beta X_{3}\right) .
$$

Thus, we have (3.30).

Now, we show necessary and sufficient conditions for a proper slant submersion of type 1 to be totally geodesic. Recall that a smooth map $\psi$ between (semi-) Riemannian manifolds $\left(N, g_{N}\right)$ and $\left(B, g_{B}\right)$ is called a totally geodesic map if $\left(\nabla \psi_{*}\right)\left(X_{1}, X_{2}\right)=0$ for all $X_{1}, X_{2} \in \Gamma(T N)$. 
Theorem 3.24. Let $\psi:\left(N, g_{N}, \varphi, \eta, \xi\right) \rightarrow\left(B, g_{B}\right)$ be a proper slant submersion of type 1 . If $N$ is a paracosymplectic manifold, at that time $\psi$ is totally geodesic if and only if

$$
g_{N}\left(h \nabla_{X_{1}} \beta \alpha X_{2}, X_{3}\right)=g_{N}\left(h \nabla_{X_{1}} \beta X_{2}, r X_{3}\right)+g_{N}\left(\mathcal{T}_{X_{1}} \beta X_{2}, t X_{3}\right)
$$

and

$$
g_{N}\left(h \nabla_{X_{4}} \beta \alpha X_{1}, X_{5}\right)=-g_{N}\left(\mathcal{A}_{X_{4}} t X_{5}+h \nabla_{X_{4}} r X_{5}, \beta X_{1}\right)
$$

for any spacelike (timelike) vector fields $X_{3}, X_{4}, X_{5} \in \Gamma\left(\left(k e r \psi_{*}\right)^{\perp}\right)$ and $X_{1}, X_{2} \in \Gamma\left(k e r \psi_{*}\right)$.

Proof. First of all, since $\psi$ is a semi-Riemannian submersion we get

$$
\left(\nabla F_{*}\right)\left(X_{4}, X_{5}\right)=0
$$

for sapacelike (timelike) vector fields $X_{4}, X_{4} \in \Gamma\left(\left(k e r \psi_{*}\right)^{\perp}\right)$.

For sapacelike (timelike) vector fields $X_{1}, X_{2} \in \Gamma\left(k e r \psi_{*}\right)$ and $X_{3}, X_{4}, X_{5} \in \Gamma\left(\left(k e r \psi_{*}\right)^{\perp}\right)$, from (2.10) and (2.12) we have

$$
\nabla_{X_{1}} X_{2}=\varphi \nabla_{X_{1}} \varphi X_{2}+\eta\left(\nabla_{X_{1}} X_{2}\right) \xi
$$

Using (2.9),(3.1) and (3.36) we get

$$
g_{B}\left(\left(\nabla \psi_{*}\right)\left(X_{1}, X_{2}\right), \psi_{*} X_{3}\right)=-g_{N}\left(\nabla_{X_{1}} \varphi \alpha X_{2}, X_{3}\right)+g_{N}\left(\nabla_{X_{1}} \beta X_{2}, \varphi X_{3}\right) .
$$

Using (3.1) and (3.2) we get

$$
\begin{aligned}
g_{B}\left(\left(\nabla \psi_{*}\right)\left(X_{1}, X_{2}\right), \psi_{*} X_{3}\right) & =-g_{N}\left(\nabla_{X_{1}} \alpha^{2} X_{2}, X_{3}\right)-g_{N}\left(\nabla_{X_{1}} \beta \alpha X_{2}, X_{3}\right) \\
& +g_{N}\left(\nabla_{X_{1}} \beta X_{2}, t X_{3}\right)+g_{N}\left(\nabla_{X_{1}} \beta X_{2}, r X_{3}\right) .
\end{aligned}
$$

Using (2.3), (2.4) and (3.3) we have

$$
\begin{aligned}
g_{B}\left(\left(\nabla \psi_{*}\right)\left(X_{1}, X_{2}\right), \psi_{*} X_{3}\right) & =-\cosh ^{2} \omega g_{N}\left(\nabla_{X_{1}} X_{2}, X_{3}\right)-g_{N}\left(h \nabla_{X_{1}} \beta \alpha X_{2}, X_{3}\right) \\
& +g_{N}\left(\mathcal{T}_{X_{1}} \beta X_{2}, t X_{3}\right)+g_{N}\left(h \nabla_{X_{1}} \beta X_{2}, r X_{3}\right) .
\end{aligned}
$$

Hence we obtain

$$
\begin{aligned}
-\sinh ^{2} \omega g_{B}\left(\left(\nabla \psi_{*}\right)\left(X_{1}, X_{2}\right), \psi_{*} X_{3}\right) & =-g_{N}\left(h \nabla_{X_{1}} \beta \alpha X_{2}, X_{3}\right)+g_{N}\left(\mathcal{T}_{X_{1}} \beta X_{2}, t X_{3}\right) \\
& +g_{N}\left(h \nabla_{X_{1}} \beta X_{2}, r X_{3}\right) .
\end{aligned}
$$

Similarly, we get

$$
\begin{aligned}
-\sinh ^{2} \omega g_{B}\left(\left(\nabla \psi_{*}\right)\left(X_{1}, X_{4}\right), \psi_{*} X_{5}\right) & =-g_{N}\left(\mathcal{A}_{X_{4}} t X_{5}+h \nabla_{X_{4}} r X_{5}, \beta X_{1}\right) \\
& -g_{N}\left(h \nabla_{X_{4}} \beta \alpha X_{1}, X_{5}\right) .
\end{aligned}
$$

Thus from (3.37) and (3.38), we get (3.34) and (3.35).

Acknowledgment. The author is grateful to the referees for their valuable comments and suggestions.

\section{References}

[1] M.A. Akyol, Conformal semi-slant submersions, Int. J. Geom. Methods Mod. Phys. 14 (7), 2017.

[2] M.A. Akyol, Conformal anti-invariant submersions from cosymplectic manifolds, Hacet. J. Math. Stat. 46 (2), 177-192, 2017.

[3] G. Baditoiu and S. Ianus, Semi-Riemannian submersions from real and complex pseudo-hyperbolic spaces, Diff. Geom. and appl. 16, 79-84, 2002.

[4] J.P. Bourguignon and H.B. Lawson, A mathematician's visit to Kaluza- Klein theory, Rend. Sem. Mat. Univ. Politec. Torino, Special Issue, 143-163, 1989.

[5] J.P. Bourguignon and H.B. Lawson, Stability and isolation phenomena for Yang-Mills fields, Comm. Math. Phys. 79, 189-230, 1981.

[6] A.V. Caldarella, On para-quaternionic submersions between para-quaternionic Kähler manifolds, Acta Applicandae Mathematicae 112, 1-14, 2010. 
[7] P. Dacko, On almost para-cosymplectic manifolds, Tsukuba J. Math. 28 (1), 193-213, 2004.

[8] M. Falcitelli, S. Ianus and A. M. Pastore, Riemannian Submersions and Related Topics, World Scientific, 2004.

[9] Y. Gündüzalp and B. Șahin, Paracontact semi-Riemannian submersions, Turk. J.Math. 37 (1), 114-128, 2013.

[10] Y. Gündüzalp, B. Șahin, Para-contact para-complex semi-Riemannian submersions, Bull. Malays. Math. Sci. Soc. 37 (1), 139-152, 2014.

[11] Y. Gündüzalp, Anti-invariant semi-Riemannian submersions from almost paraHermitian manifolds, J. Funct. Spaces 2013, ID 720623, 2013.

[12] Y. Gündüzalp, Slant submersions from almost product Riemannian manifolds, Turk. J. Math. 37, 863-873, 2013.

[13] Y. Gündüzalp and M.A. Akyol, Conformal slant submersions from cosymplectic manifolds, Turk. J. Math. 42 (5), 2672-2689, 2018.

[14] A. Gray, Pseudo-Riemannian almost product manifolds and submersions, J. Math. Mech. 16, 715-737, 1967.

[15] S. Ianus and M. Visinescu, Kaluza-Klein theory with scalar fields and generalised Hopf manifolds, Classical Quantum Gravity 4, 1317-1352, 1987.

[16] S. Ianus, and M. Visinescu, Space-time compactification and Riemannian submersions, The mathematical heritage of C.F. Gauss, World Sci. Publ., River Edge, NJ, 358-371, 1991.

[17] S. Ianus, R. Mazzocco and G. E. Vilcu, Riemannian submersions from quaternionic manifolds, Acta Appl. Math. 104, 83-89, 2008.

[18] S. Ianus, G.E. Vilcu and R.C. Voicu, Harmonic maps and Riemannian submersions between manifolds endowed with special structures, Banach Center Publications 93, 277-288, 2011.

[19] I.K. Erken and C. Murathan, On slant Riemannian submersions for cosymplectic manifolds, Bull. Korean Math. Soc. 51 (6), 1749-1771, 2014.

[20] B. O'Neill, The fundamental equations of a submersion, Michigan Math. J. 13, 459469, 1966.

[21] B. O'Neill, Semi-Riemannian Geometry with Application to Relativity, Academic Press, New York, 1983.

[22] K.S.Park, H-slant submersions, Bull. Korean Math. Soc. 49, 329-338, 2012.

[23] B. Șahin, Slant submersions from almost Hermitian manifolds, Bull. Math. Soc.Sci. Math. Roumanie Tome. 54, 93-105, 2011.

[24] B. Șahin, Anti-invariant Riemannian submersions from almost Hermitian manifolds, Central European J.Math 8 (3), 437-447, 2010.

[25] H.M. Taștan , B. Șahin and Ș. Yanan, Hemi-slant submersions, Mediterr. J. Math. 13, 2171-2184, 2016.

[26] J. Welyczko, On Legendre curves in 3-dimensional normal almost paracontact metric manifolds, Results Math. 54, 377-387, 2009.

[27] B. Watson, Almost Hermitian submersions, J. Differential Geom. 11, 147-165, 1976.

[28] S. Zamkovoy, Canonical connections on paracontact manifolds, Ann. Global Anal. Geometry 36, 37-60, 2009. 